\title{
Walther, Jörg
}

Kindertageseinrichtungen und Fachberatung im Wandel

Hense, Margarita [Hrsg.]: Fachberatung für Kindertageseinrichtungen. Erfolgschancen erhöhen. Göttingen : Vandenhoeck \& Ruprecht 2010, S. 91-108. - (Frühe Bildung und Erziehung )

Quellenangabe/ Reference:

Walther, Jörg: Kindertageseinrichtungen und Fachberatung im Wandel - In: Hense, Margarita [Hrsg.]: Fachberatung für Kindertageseinrichtungen. Erfolgschancen erhöhen. Göttingen : Vandenhoeck \& Ruprecht 2010, S. 91-108 - URN: urn:nbn:de:0111-opus-30710 - DOI: 10.25656/01:3071

\section{Nutzungsbedingungen}

Gewährt wird ein nicht exklusives, nicht übertragbares, persönliches und beschränktes Recht auf Nutzung dieses Dokuments. Dieses Dokument ist ausschließlich für den persönlichen, nicht-kommerziellen Gebrauch bestimmt. Die Nutzung stellt keine Übertragung des Eigentumsrechts an diesem Dokument dar und gilt vorbehaltlich der folgenden Einschränkungen: Auf sämtlichen Kopien dieses Dokuments müssen alle Urheberrechtshinweise und sonstigen Hinweise auf gesetzlichen Schutz beibehalten werden. Sie dürfen dieses Dokument nicht in irgendeiner Weise abändern, noch dürfen Sie dieses Dokument für öffentliche oder kommerzielle Zwecke vervielfältigen, öffentlich ausstellen, aufführen, vertreiben oder anderweitig nutzen.

Mit der Verwendung dieses Dokuments erkennen Sie die Nutzungsbedingungen an.

\section{Terms of use}

We grant a non-exclusive, non-transferable, individual and limited right to using this document

This document is solely intended for your personal, non-commercial use. Use of this document does not include any transfer of property rights and it is conditional to the following limitations: All of the copies of this documents must retain all copyright information and other information regarding legal protection. You are not allowed to alter this document in any way, to copy it for public or commercial purposes, to exhibit the document in public, to perform, distribute or otherwise use the document in public.

By using this particular document, you accept the above-stated conditions of use.

\section{Kontakt / Contact:}

peDOCS

DIPF | Leibniz-Institut für Bildungsforschung und Bildungsinformation

Informationszentrum (IZ) Bildung

E-Mail: pedocs@dipf.de

Internet: www.pedocs.de

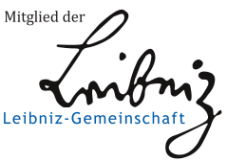




\section{Jörg Walther}

\section{Kindertageseinrichtungen und Fachberatung im Wandel}

Kindertageseinrichtungen sind vielfältigen Veränderungsprozessen unterworfen, in denen Fachberatung eine Schlüsselrolle zukommt. Bevor überhaupt über Effizienz nachgedacht werden kann, gilt es, diese Veränderungen und deren Auswirkungen auf Fachberatung zu beschreiben. Dies geschieht in diesem Beitrag am Beispiel der Evangelischen Tageseinrichtungen für Kinder in der Evangelischen Kirche im Rheinland und der Diakonie Rheinland-Westfalen-Lippe e. V. mit besonderem Bezug zur aktuellen Landesgesetzgebung in NordrheinWestfalen. Abschließend sind erste Überlegungen zu einer Effizienzdarstellung formuliert.

\section{Kindertageseinrichtungen im Wandel}

Ohne Zweifel befinden sich Tageseinrichtungen für Kinder und andere Formen der Tagesbetreuung in einer Phase des massiven Umbruchs und enormer Veränderungen. Vier Hauptströmungen zeichnen diesen Wandel entscheidend mit.

Wissen und die aus ihm entstehenden Kompetenzen in einer globalen Welt bilden ein existentielles und ethisch fundiertes Zukunftspotenzial. „Die Kinder von heute sind die Werteträger von morgen“ (Tries, 2009, S. 15). Dieser uns alle bekannte Zusammenhang hat, nicht zuletzt auch durch vielfältige Ergebnisse der Kognitionswissenschaften (vgl. Laewen 2002), zu einem veränderten Bild frühkindlicher Betreuung, Bildung und Erziehung geführt. In diesen Ergebnissen ist Bildung zum Antreiber geworden, mit der Chance, Bildung als Sprache der Freiheit zu gestalten. Die Bundesländer haben vor diesem Hintergrund Bildungspläne, Bildungsvereinbarungen und Erziehungsempfehlungen entwickelt, die sich in vielen Fällen in einem ersten Erprobungs- und Bewährungszustand befinden. Damit werden die Tageseinrichtungen für Kinder auf der einen Seite als Bildungsinstitution aufgewertet, sie müssen sich auf der anderen Seite aber auch diesem Anspruch stellen.

Die gesellschaftliche Rolle von Familie hat sich in den letzten Jahrzehnten stark gewandelt. Zugespitzt formuliert leben wir gerade in den Ballungsgebieten in einem multikulturellen Patchwork verschiedenster religiöser und ander- 
weitig ausgeprägter Anspruchswelten. In diesem Umfeld haben in nicht wenigen Fällen Eltern bzw. Elternteile ihre Erziehungsorientierung verloren, die sie unter anderem in den Tageseinrichtungen für Kinder und Schulen wieder zu finden hoffen. Zudem verjüngt sich infolge der politisch initiierten Vereinbarkeit von Familie und Beruf die Alterspanne in den Tageseinrichtungen auf Kinder unter drei Jahren, unterstützt durch die bundespolitische aktuelle Veränderung und Gewährung eines Rechtsanspruchs ab Vollendung des ersten Lebensjahres, gültig ab dem Jahr 2013. Am oberen Ende der Altersskala werden Familien mit ihren Kindern zunehmend durch eine frühere Einschulung, ab dem 5. Lebensjahr, mehr in die Entscheidung der Schulbildung gedrängt. In der Vergangenheit ist die Altersspanne der Hortkinder (6-12 Jahre) als Konsequenz der offenen Ganztagsschulen in vielen Landesbereichen komplett gebrochen.

Es wird dabei deutlich, dass die Diskussion um die frühere Einschulung jetzt erst richtig begonnen hat, insbesondere im Hinblick auf die Unterschiedlichkeit der Angebote in Qualität und Quantität im europäischen Vergleich.

Die ersten konzeptionellen Antworten hierauf sind vermeintlich neue Angebotsformen z.B. der Familienzentren in Nordrhein-Westfalen sowie die einzelne Tageseinrichtung als übergreifendes Versorgungssystem für Notsituationen: Kinder sollen ggf. bis zu 24 Stunden am Tag betreut oder in (leichteren) Krankheitsfällen auch rund um die Uhr versorgt und behütet werden können.

Diese Erwartungen an ein einrichtungsübergreifendes Versorgungs- und Angebotssystem im systemischen Ansatz gelten inzwischen als zukünftige Minimalforderungen gegenüber weit extensiveren Erwartungen von Politik und Gesellschaft, die Antworten auf die Situation suchen, dass Familien und ihre Kinder im gängigen Hilfesystem nicht mehr nachhaltig erreicht werden können. Neuere Entwicklungen in den gesetzlichen Regelungen werden nach und nach diese veränderten Bedingungen unterstützen.

Hiermit verlässt die Kindertageseinrichtung ihren pädagogischen Rahmen und erweitert ihre sozialpädagogischen Herausforderungen in Form von Familien- und Elternberatung, -unterstützung und/oder besonderer Beobachtung des Kindeswohls. Ein Drahtseilakt kann es sein, wenn dieses Kindeswohl im Spannungsbogen zwischen Elterninteressen und dem gelingendem Aufwachsen von Kindern zu sichern und zu unterstützen ist.

Auch Tageseinrichtungen für Kinder stehen im Dauerfokus internationaler Vergleichbarkeit. Die Studie „Starting Strong“ (2004) oder auch „Baby-Pisa“ genannt, lobt den ganzheitlichen Ansatz frühkindlicher Betreuung, Bildung und Erziehung. Diese weist aber auch aus, dass die fachliche Qualifikation sowie die Qualitätsunterschiede zwischen den Tageseinrichtungen für Kinder sehr unterdurchschnittlich ausfallen und ein Gefälle mit Höhen und Tiefen in der Ausgestaltung der Qualität abbilden. Hieraus ergeben sich wesentliche und verschiedene Konsequenzen, die weiter unten im Text ausgeführt werden. 


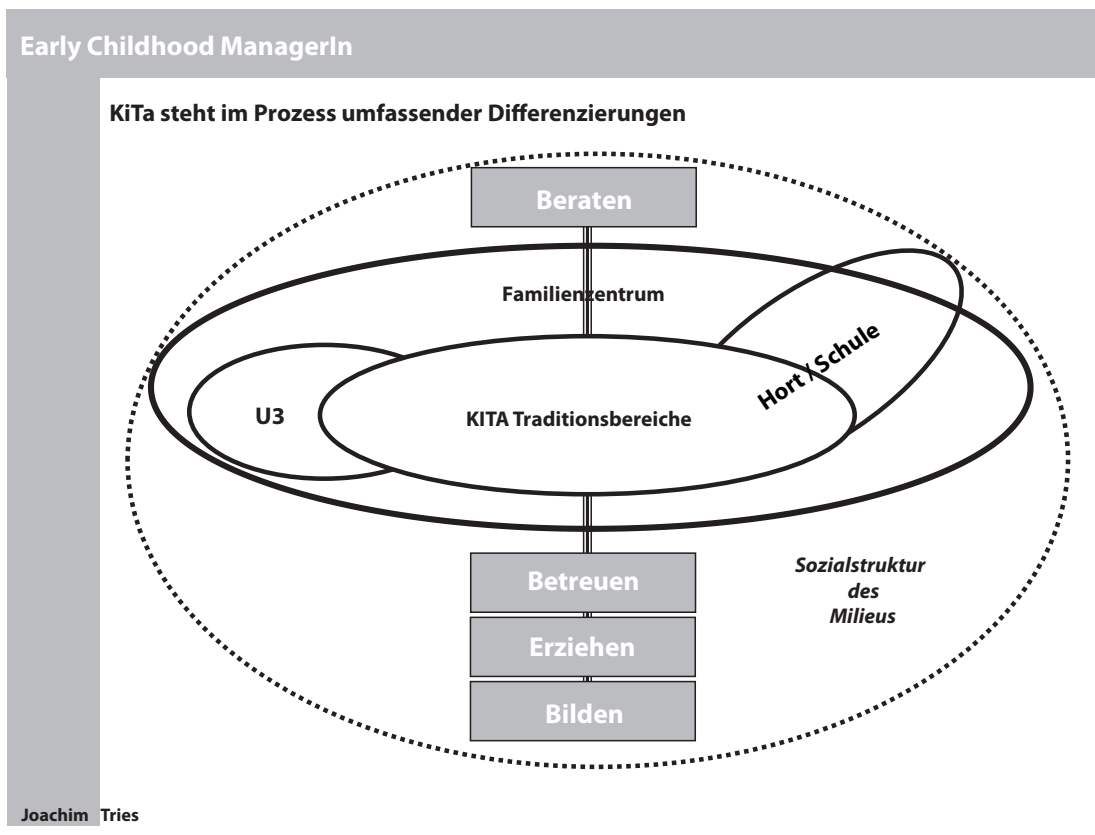

Abb. 1: Vertikale und horizontale Differenzierung der Tageseinrichtungen für Kinder (Tries 2007)

Obwohl die Anforderungen an Tageseinrichtungen für Kinder vielschichtiger und anspruchsvoller geworden sind, stehen auch zukünftig weiterhin unzureichende Finanzmittel zur Verfügung. Der gesellschaftliche Anspruch bzw. die Erwartungen und die Bereitstellung und Abbildung der Finanzierungssysteme für Tageseinrichtungen für Kinder, drohen weiter auseinanderzugehen.

\begin{tabular}{|c|c|c|c|}
\hline Land & $\begin{array}{l}\text { Gesetzliche } \\
\text { Änderungen }\end{array}$ & Konsequenzen & Anmerkungen \\
\hline NRW & KiBiZ (2008) & $\begin{array}{l}\text { Jährlich veränderte Kind- } \\
\text { pauschalen - Zusammen- } \\
\text { setzung statt anteiligem, } \\
\text { festen Betriebskostenzu- } \\
\text { schusssystem }\end{array}$ & $\begin{array}{l}\text { keine langfristige Sicherheit } \\
\text { der Refinanzierung, nur } \\
\text { schrittweise Anpassung } \\
\text { an Veränderungen, } \\
\text { Kommunalisierung }\end{array}$ \\
\hline $\begin{array}{l}\text { Rheinland- } \\
\text { Pfalz }\end{array}$ & $\begin{array}{l}\text { zu erwarten } \\
\text { in } 2011\end{array}$ & $\begin{array}{l}\text { Umstellung auf komplett } \\
\text { neues Finanzierungssystem } \\
\text { statt anteiligem, jährlichen } \\
\text { festen Betriebskostenzu- } \\
\text { schusssystem }\end{array}$ & $\begin{array}{l}\text { Trägerverbände sollen } \\
\text { in die Ausgestaltung } \\
\text { des System einbezogen } \\
\text { werden }\end{array}$ \\
\hline
\end{tabular}




\begin{tabular}{|c|c|c|c|}
\hline Land & $\begin{array}{l}\text { Gesetzliche } \\
\text { Änderungen }\end{array}$ & Konsequenzen & Anmerkungen \\
\hline Saarland & KBBG (2008) & $\begin{array}{l}\text { Erstmalig im Detail fest- } \\
\text { gezurrtes System: Darstel- } \\
\text { lung der Verantwortung } \\
\text { und Aufgabenbeschrei- } \\
\text { bung, zu erwartende Ver- } \\
\text { änderung durch neue } \\
\text { Regierung im Elementar- } \\
\text { und Schulbereich }\end{array}$ & $\begin{array}{l}\text { Landesweite Rechtsverord- } \\
\text { nungen klären nach und } \\
\text { nach Detail-fragen in enger } \\
\text { Rückkopplung mit den } \\
\text { Trägerorganisationen, } \\
\text { schrittweise Anpassung }\end{array}$ \\
\hline Hessen & $\begin{array}{l}\text { Mindestvor- } \\
\text { aussetzungs- } \\
\text { verordnung } \\
2008 / 2009\end{array}$ & $\begin{array}{l}\text { Personalbemessungs- } \\
\text { system ist in der aktuellen } \\
\text { Überprüfung, eventuelle } \\
\text { Veränderung durch neue } \\
\text { Regierung }\end{array}$ & $\begin{array}{l}\text { Kommunalisierung, } \\
\text { Ausgestaltung vor Ort, } \\
\text { wenig umgesetzte } \\
\text { landesweite verbindliche } \\
\text { Standards }\end{array}$ \\
\hline
\end{tabular}

Tab. 1: Änderungen der Rahmenbedingungen für Kindertageseinrichtungen am Beispiel ausgewählter Bundesländer (Einzugsgebiet des Rheinischen Verbandes)

Finanzierungsmodalitäten und eine weitere Professionalisierung können sich auch auf Trägerstrukturen (zumindest der Kirchen und der freien Wohlfahrtspflege) auswirken, wie dies beispielhaft am Kinderbildungsgesetz und seinen Ausführungsverordnungen aufgezeigt wird. Diese Veränderungen wiederum strahlen auf die Aufgabenanforderungen und auf das Rollenverständnis von Fachberatung aus.

Generell führt die Aufnahme von Kindern unter drei Jahren (U3) zu veränderten Gruppenstrukturen. Die Ausweitung der Angebotsstruktur mit umfassenden Umbaumaßnahmen, der Entwicklung neuer pädagogischer Konzepte, hierauf aufzubauender Bildungsangebote für die Mitarbeitenden sowie erhöhter Erwartungen an die fachliche Eingangsqualifikation des Personals sind weitere Baustellen für Fachberatung. So sollen in NRW Kinderpflegerinnen und fachpädagogisch nicht ausgebildete Ergänzungskräfte im Regelfall in Gruppen, die Kinder unter drei Jahren aufnehmen, nicht mehr refinanziert werden.

Das Kinderbildungsgesetz bzw. seine Ausführungsbestimmungen verorten den Bildungsanspruch für Kinder in Tageseinrichtungen. Anforderungen in pädagogisch fernen Bildungsfeldern, z. B. naturwissenschaftlichen, mathematischen und sprachlichen Aufgabenfeldern, erfordert ein neues Bildungsverständnis, das über die herkömmlichen Fort- und Weiterbildungsmaßnahmen hinausgeht.

Landespolitisches Ziel ist es ferner, bis ins Jahr 2012 ca. 3000 Kindertageseinrichtungen zu Familienzentren auszubauen. Grundgedanke dabei ist, vernetzte Angebote für Kinder und Eltern im Quartiersnetzwerk zur Verfügung zu stel- 
len. Hieraus ergeben sich wieder für Fachberatung in der Vermittlung verschiedene Konsequenzen. Nicht nur müssen funktionstüchtige Netzwerke auf- oder ausgebaut werden, sondern auch das Verständnis und die Haltung in der Arbeit mit Eltern und Familien grundlegend überdacht und in systemische, ganzheitliche Verantwortung weiterentwickelt werden. Die Leiterinnen dieser neuen Familienzentren stehen nach kurzer Zeit in einem intensiven Rollenwechsel von der pädagogisch fundierten hin zur managementorientierten Führung der Einrichtung, einer Aufgabe, auf die sie nicht oder in Teilen nur mangelhaft vorbereitet sind.

Schließlich hat die Qualitätsentwicklung nach Gütesiegeln oder in Anlehnung an die DIN EN ISO 9001:2008 auch Träger und Tageseinrichtungen für Kinder erreicht. Im Einzugsbereich der Rheinischen Kirche bereiten sich aktuell ca. $40 \%$ der evangelischen Tageseinrichtungen für Kinder auf diese Entwicklung mit einer entsprechenden Maßnahme für die Erreichung eines Qualitäts- und Gütesiegel vor. Die Bereitschaft, sich auf eine Qualitätszertifizierung einzulassen, verstärkt dabei die Frage, ob vor dem Hintergrund der oben beschriebenen inhaltlichen und finanziellen Differenzierungen überhaupt noch kleine Träger in der Lage sind, Einrichtungen zu führen und den Qualitätsprozessen, die sich an den Träger richten, entsprechen zu können. Infolgedessen verändert sich die Trägerlandschaft und Träger werden sich zu Qualitätsnetzwerken oder sogar weiterhin zu neuen örtlichen, großen Trägerorganisationen zusammenschließen, wie es im Laufe des letzten Jahres in den Ballungsgebieten Düsseldorf, Wuppertal und Köln bereits passiert ist.

Fazit: Der umfassende Wandel, dem Tageseinrichtungen für Kinder ausgesetzt sind, wirkt sich auf alle Akteure aus, die für diese Einrichtungen Verantwortung tragen. Dies betrifft nicht nur das fachpädagogische Personal, sondern auch Trägeraufgaben sowie in bedeutender Art und Weise die Metaebene der Fachberatung. Dabei sind weniger die Veränderungen selbst das Problem, sondern eher das Phänomen, diesen Wandel nicht ganzheitlich zu erfassen, um darauf strategisch antworten zu können. Vielerorts lassen sich Tageseinrichtungen in einem regionalen Markt erfassen, in dem die Marktakteure regionale Antworten zu finden versuchen. Es gibt keine flächendeckende Entwicklung, geschweige denn eine Strategie, noch politisch zur Verfügung gestellte finanzielle Mittel, um auf die Herausforderungen und Risiken zu reagieren. Systemische und ganzheitliche Antworten müssen gefunden werden um den Systemumbruch als Chance zu begreifen. 


\section{Kompetenzausbau als wesentlicher Aspekt der Personalentwicklung}

Als Folge des beschriebenen Wandels stehen Verantwortungsträger und das pädagogische Fachpersonal vor Herausforderungen, die ohne eine systematische Fort- und Weiterbildung kaum zu bewerkstelligen sind.

Die insgesamt hohe Fort- und Weiterbildungsbereitschaft der pädagogischen Mitarbeitenden in den Kindertageseinrichtungen täuscht nicht über die Notwendigkeit hinweg, lebenslanges Lernen am und außerhalb des Arbeitsplatzes verstärkt wahrnehmen zu müssen. Die Fort- und Weiterbildungsinhalte ergeben sich nicht mehr ausschließlich aus persönlichen Neigungen, sondern zunehmend aus den oben skizzierten Differenzierungen und den jeweiligen konzeptionellen Entwicklungen der einzelnen Einrichtungen. Fort- und Weiterbildung wird zunehmend zum Bestandteil der Führungsaufgaben in einer Einrichtung und versteht sich als ein Prozess, der aus veränderten Rahmen- und Organisationsbedingungen abgeleitet wird.

Leider stehen enge Personalkontingente und knappe Finanzmittel dieser Entwicklung scheinbar gegenüber. Insofern sind Bildungssysteme zu gestalten, die den erforderlichen Bildungsbedarf aufgreifen und ökonomisch darstellen. Die schwerpunktmäßige lokale oder nur regionale Verankerung des Qualifizierungsmanagements widerspricht einer strategisch verantworteten Personalentwicklung, zu der im Kern auch die Kompetenzentwicklung der Mitarbeitenden und Verantwortungsträger gehört.

Um diesen Spannungsbogen konstruktiv zu nutzen, hat die Evangelische Kirche im Rheinland, das Diakonische Werk der Evangelischen Kirche im Rheinland e.V. und der Rheinische Verband Evangelischer Tageseinrichtungen für Kinder e. V. das „Integrierte Bildungssystem Evangelischer Kindertageseinrichtungen (IBEK) im Jahr 2008 entwickelt“. Es adressiert für alle Akteurinnen und Akteure rund um die Kindertageseinrichtungen ein aufeinander abgestimmtes Bildungssystem (vgl. auch Walther, 2009, S. 4ff). Die Bildungsinhalte werden durch Expertinnen und Experten-Gremien, in denen Fachberatung wiederum eine gravierende Schlüsselrolle zukommt, definiert, um sodann in Curriculumsgruppen ausgestaltet zu werden. Hierdurch werden Standards für Inhalte und Didaktiken gesichert.

Lernen im Rahmen dieser Bildungsmodule findet nur noch zu ca. $30 \%$ an einem Präsenzort und zu jeweils weiteren $30 \%$ in Form von Projektaufgaben, Selbststudium sowie moderiertem Studium statt. Den Teilnehmerinnen stehen dabei verschiedene Unterstützungssysteme wie Lerngruppen oder gar virtuelle Lernmethoden zur Verfügung.

Der erfolgreiche Abschluss einer Fort- oder Weiterbildungsmaßnahme wird durch einen Leistungsnachweis dokumentiert. Das Spektrum reicht von Projektberichten über Klausuren bis zu Kolloquien. Hierdurch sichern die Projekt- 


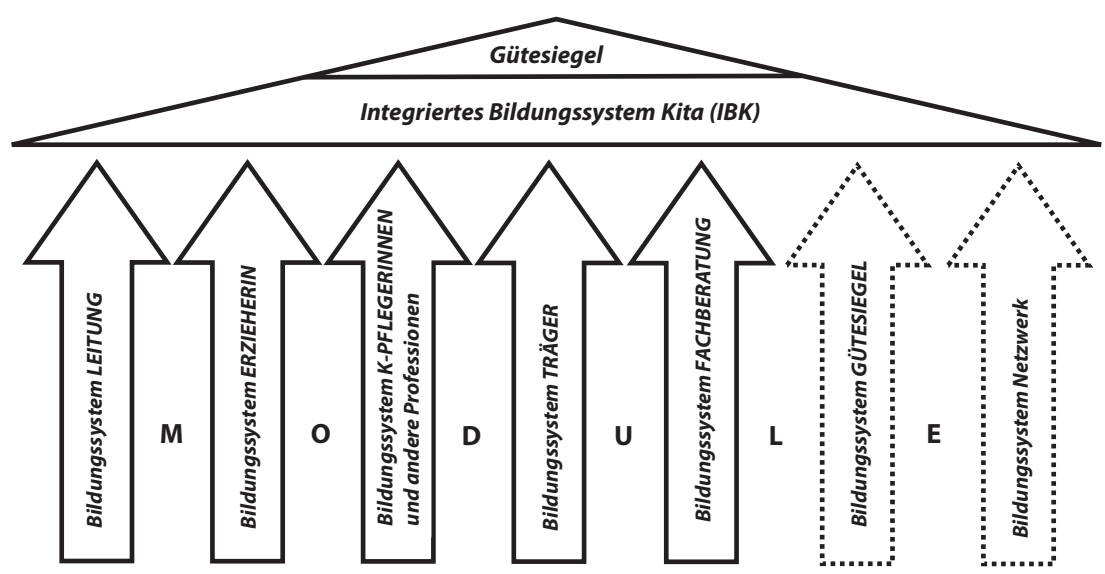

EVANGELISCH

PRO KIND PRO FREIHEIT

GESTALTEN IST SELBSTGESTALTEN

\section{QUALITÄT IN DER VIELFALT}

Abb. 2: Das integrierte Säulenprinzip von IBEK

räger in IBEK den immer wichtiger werdenden Anspruch ab, früheres Lernen in zukünftige Maßnahmen anerkannt zu bekommen (Accreditation of Prior Learning, APL). Den Teilnehmerinnen können frühere Fortbildungen durch IBEK anerkannt werden, soweit sie inhaltlich und umfänglich mit den Bildungsmaßnahmen nach IBEK übereinstimmen. Diese Durchlässigkeit in horizontaler und vertikaler Weise betrachtet IBEK als Ressourcen schonendes und auf Nachhaltigkeit angelegtes System.

Gegenseitige Anerkennungen können bis in die Hochschulen hineinreichen. Insofern werden die Bildungsmodule und deren Arbeitsaufwand gemäß der „Richtlinien nach Bologna“ organisiert. Zentrale Berechnungsgröße ist der sogenannte Credit Point, nach dem sich Hochschulen in der Organisation ihrer Studiengänge ausrichten. Ein Credit Point umfasst zwischen 25 und 30 Vollzeitstunden Lernaufwand, unabhängig von seinem Lernort.

Diese bildungsbiografische Durchlässigkeit ist auch vor dem realen Hintergrund zwingend erforderlich, wirksame Antworten auf die Akademisierungsdebatte zu eröffnen. Mit zunehmender Bedeutsamkeit der Kindertageseinrichtungen wird ein entsprechendes Kompetenzprofil zu fordern sein und dies bedeutet, das pädagogische Personal in Anteilen (z. B. für Fachberatung und in der Leitungsfunktion) verbindlich zu akademisieren. Einem durch diese Debatte ausgelösten Kompetenzwettbewerb auf allen beruflichen Ebenen der Ta- 
geseinrichtungen werfen allerdings auf drei Ebenen entscheidende und verschiedene Aspekte auf:

1. Die Ausbildung zur staatlich anerkannten Erzieherin dauert zwischen vier und fünf Jahre in einer attraktiven Kombination aus Theorie und Praxis. In dieser Phase wird ein Wissens- und Könnensschatz aufgebaut, der ein akademisches Studium kaum zu erbringen mag.

2. Auch wenn bundesweit mittlerweile ca. 60 Institute Bachelorausbildungen unterschiedlich berufsbegleitend anbieten, kann hierdurch der akute Kompetenzbedarf in den nächsten Jahren nicht gedeckt werden (vgl. König \& Pasternak, 2008).

3. Als Forderung steht daher folgerichtig ein Bildungssystem im Raum, das auf den Erfahrungsschatz aufbaut und einen entsprechenden Bachelor-Abschluss bedient.

Entscheidend in diesem Kontext ist es, vorhandene Kompetenz zu nutzen und zu fördern und vor allem anzuerkennen. Dies ist nicht selbstverständlich. Einen berufspolitischen schwierigen Sachverhalt erlaubte sich das Bundesland Nordrhein - Westfalen in seinen Personalverordnungen 2008 infolge des Kinderbildungsgesetzes. Praxiserfahrene Mitarbeitende, die nicht über eine Ausbildung als Erzieherin verfügten bzw. für diese sie sich bis zum Stichtagsjahr 2013 angemeldet hätten, sollten im Regelfall von der Refinanzierung der zukünftig dominierenden Gruppenformen mit Kindern unter drei Jahren ausgeschlossen werden. Die stringente Umsetzung dieses anfänglichen Vorhabens hätte fachpolitisch, arbeitsmarktpolitisch und auch menschliche schwierige Verhältnisse für die Arbeitsebenen der Tageseinrichtungen für Kinder ausgelöst. Träger hätten praxiserfahrene und wertvolle Mitarbeiterinnen (aufgrund des Qualifizierungsniveaus und biografischer Bedingungen) bis in die Arbeitslosigkeit entlassen müssen. In den nächsten Jahren wird allerdings durch die oben genannten Differenzierungsprozesse mehr Personal benötigt, als der Arbeitsmarkt anbieten kann. In verschiedenen Regionen herrscht gegenwärtig bereits ein erkennbarer Mangel an Fachkräften und damit schwierige Situationen für die Verantwortung von Trägern und Fachberatung.

Fazit: Kindertageseinrichtungen als familiales und volkswirtschaftliches $\mathrm{Kal}$ kül stehen in einer Kompetenzoffensive, die mit herkömmlichen Instrumenten kaum zu bewerkstelligen ist. Fachberatung nimmt darin eine nicht mehr lokale, sondern eher eine strategische bildungspolitische Aufgabe und für den Träger entlastende Mitsteuerung in der Personalverantwortung wahr. 


\section{Evangelische Qualitätsentwicklung}

Die Vielfalt der Profile und Konzepte der Tageseinrichtungen für Kinder, auch das große Engagement, mit dem sie gelebt werden, täuschen nicht über erkennbare Qualitätsschwankungen zwischen den Einrichtungen hinweg (Starting Strong). Die aktuelle Debatte, nach der Tageseinrichtungen ihre Qualität gemäß der Qualitätsnorm ISO 9000 bzw. an ein danach angelehntes Siegel nachweisen sollen, eröffnet im Gesamtkontext des Wandels mehr Chancen als Risiken: Die neuerlichen Qualitätsoffensiven liefern entscheidende Impulse, Qualitätsaussagen zu hinterfragen, ggf. zu verbessern und als Standards festzuschreiben.

Das aus der Industrie entlehnte Qualitätsverständnis bedarf in Tageseinrichtungen für Kinder einer grundlegenden Verankerung. „Die Umsetzung großer Leitsätze braucht eben viele kleine Alltagsentscheidungen, um ein Profil deutlich zu machen“ (Blank, 2009). Der Grundstein eines rheinisch- evangelischen Verständnisses von Qualitätsarbeit fußt in dem Kern, Kindern insbesondere eine Hoffnung auf ihre Zukunft vermitteln zu wollen (vgl. Rheinischer Verband e. V. 2002). Dieses Verständnis von Qualitätsmanagement basiert auf dialogischer, prozesshafter Haltung und Handlung.

Gegenwärtig stehen den Trägern der Evangelischen Kirche zwei Qualitätsmodelle alternativ oder additiv zur Verfügung. Die damit verbundenen Qualitätsnormen und deren Aufwendungen fallen unterschiedlich aus.

Ungeachtet der Einzelentscheidung, welcher Weg nun der angemessenere sei, müssen Träger/Qualitätsverbünde und Fachberatung bereit sein, sich verbindlich auf diesen Prozess einzulassen und dem Beauftragten für Qualität bzw.

\begin{tabular}{|l|l|l|}
\hline & $\begin{array}{l}\text { Evangelisches Gütesiegel } \\
\text { nach BETA }\end{array}$ & $\begin{array}{l}\text { Zertifizierung nach } \\
\text { DIN IS0 9001:2009 }\end{array}$ \\
\hline Anzahl der Prozesse & $\begin{array}{l}\text { 19 Führungs-, 19 Kern-, } \\
1 \text { Unterstützungsprozess }\end{array}$ & $\begin{array}{l}\text { plus } \\
17 \text { Führungs-, 0 Kern-, } \\
3 \text { Unterstützungsprozesse }\end{array}$ \\
\hline Zertifizierer & Rheinischer Verband > IBEK & $\begin{array}{l}\text { akkreditiertes Unternehmen } \\
\text { mit Rahmenvertrag }\end{array}$ \\
\hline Rezertifizierung & alle 5 Jahre & alle 3 Jahre \\
\hline Auditorlnnen & Intern qualifiziert & $\begin{array}{l}\text { plus } \\
\text { externe Qualifizierung }\end{array}$ \\
\hline
\end{tabular}

Abb. 3: Entscheidung für ein Qualitätssystem und Folgen 
der beauftragten Fachberatung ein administratives Durchgriffsrecht gewähren. Darin werden nicht nur pädagogische Kernprozesse, sondern auch trägerverantwortliche Führungs- und Unterstützungsprozesse mit nachhaltigen Folgen $\mathrm{zu}$ formulieren sein.

Entscheidend und wesentlich für diese Betrachtung ist natürlich die Rolle der Fachberatung in diesem Prozess. Als Bindeglied zwischen Träger und Einrichtung kommt dieser Position eine besondere Schanierfunktion zu, da die verschiedenen Prozesse in Bezug auf Einrichtung und Träger aufeinander abgestimmt und verbindlich vereinbart werden müssen. Das derzeitige Spektrum, in dem Fachberatung diese Aufgabe wahrnimmt oder diese Entwicklung auf der Metaebene begleitet und den Qualitätsbeauftragten auf Augenhöhe unterstützt, ist im rheinischen Bereich relativ groß.

\section{Fachberatung als Qualitätsmanagementbeauftragte (QMB)}

In kleineren Verbundsystemen kann Fachberatung die Rolle einer Qualitätsbeauftragten übernehmen. Hierfür bedarf es einer besonderen Qualifizierung und Rollenklärung zwischen Beratung und der Leitungsfunktion von Fachberatung, denn die Beratung wäre nicht mehr auf Anfrage gestaltet, sondern mit Verbindlich- und Nachhaltigkeiten ausgestattet.

\section{Fachberatung als Moderatorin}

Die Qualitätsentwicklung richtet sich im Wesentlichen an pädagogischen und führungsbezogenen Prozessen aus. Pädagogische Prozesse qualifiziert zu beschreiben, ist in der Regel geübte Praxis (Konzeptionsarbeit) für Fachberatung. Demgegenüber ist die explizite Ausgestaltung der Führungsprozesse in der Abgrenzung zwischen Träger und Einrichtung eine mehr oder minder ungewohnte Praxis. Hier kann Fachberatung eine vermittelnde und entscheidend mitgestaltende Rolle einnehmen.

\section{Fachberatung als Anbieter/Multiplikator für unterstützende Fort-/Weiterbildungsmaßnahmen}

Soll Qualität nachhaltig gelebt werden, sind unterstützende Fort- und Weiterbildungsangebote, insbesondere auch vor dem Hintergrund der sich vertikal und horizontal differenzierenden Tageseinrichtungen für Kinder angezeigt. Im IBEK wird diese differenzierte Maßnahme von Fachberaterinnen verbindlich mit verantwortet. 


\begin{tabular}{|l|l|l|}
\hline Bildungsmaßnahme & Dauer & Primäre Zielgruppen \\
\hline $\begin{array}{l}\text { Hoffnung Leben im } \\
\text { Qualitätsprozess }\end{array}$ & 2 Tage & Leiterlnnen \\
\hline Einführung in QM & 2 Tage & $\begin{array}{l}\text { Leiterlnnen, Träger, } \\
\text { Fachberatung }\end{array}$ \\
\hline Leiten und steuern & $8 * 2$ Tage & $\begin{array}{l}\text { Leiterlnnen, } \\
\text { QM-Verantwortliche }\end{array}$ \\
\hline Träger - Crashkurse & Voraussichtlich $4 * 2$ Tage & Träger \\
\hline
\end{tabular}

Abb. 4: Fortbildungsbausteine für ein evangelisches Qualitätsmanagement

Diese Module wurden durch sogenannte Curriculumsgruppen erarbeitet, an denen Fachberatung entscheidend beteiligt waren. Zudem wirken sie regional als Dozentinnen bzw. als Lernortmoderatorinnen aktiv mit.

\section{Fachberatung als interne Auditorin}

Maßnahmen im Qualitätsmanagement schließen mit einer Überprüfung der Qualitätsprozesse und -standards (Audit) in Tageseinrichtungen für Kinder ab: Der positive Ausgang des Audits führt zur Vergabe eines Gütesiegels bzw. Zertifikats. Solche Audits müssen nicht nur eingeübt werden, sie sollten auch jährlich als „interne“ Überprüfungen durchgeführt werden. Bei Erlangung eines Gütesiegels nach DIN-ISO-Kriterien müssen diese Audits verbindlich jährlich durchgeführt werden.

Hier erhält Fachberatung wieder die Möglichkeit, eine solche Funktion zu übernehmen und die Verbindlichkeit der zu vereinbarenden Verbesserungen und damit die Qualität der Tageseinrichtung für Kinder zu erhöhen. Natürlich bedarf es hierfür entsprechender Qualifikationen und Schulungen.

Fazit: Die Professionalisierung der Tageseinrichtungen für Kinder schreitet weiter voran und erfordert differenzierte Kompetenzen, der sich auch Fachberatung stellen und zugleich diese erworbenen Kompetenzen einbringen muss. Qualitätsmanagement stellt dabei ein wichtiges Verfahren dar. 


\section{- Selbstbild der Fachberatung}

Für den Bereich der Evangelischen Kirche im Rheinland beschreiben die Fachberaterinnen in ihrem Qualitätshandbuch 2004 fünf zentrale, als Schwerpunktthemen ausgewiesene Handlungsfelder ihrer Arbeit:

1. Beratung in der Fachberatung

2. Fortbildung in der Fachberatung,

3. Leiterinnenkonferenzen, Arbeitskreise/Arbeitsgemeinschaften und Informationsweitergabe,

4. Interne Strukturen/Arbeitskreis Fachberatung sowie

5. Öffentlichkeitsarbeit.

Dabei verstehen sie sich als Interessenvertreterinnen „... von Kindertageseinrichtungen in Politik und Kirche, sodass förderliche Rahmenbedingungen für das Aufwachsen von Kindern und deren Familien erhalten bleiben und geschaffen werden“.

\section{Beratung in der Fachberatung}

Das Handbuch weist neun Beratungsfelder aus:

1. Pädagogische Beratung

2. Konzeptionsberatung

3. Beratung zur Teamentwicklung

4. Leitungsberatung

5. Strategieberatung

6. Beratung in Konfliktsituationen

7. Beratung zur Personalentwicklung

8. Bauberatung

9. Trägerberatung

Deutlich wird hier die besondere Betonung einrichtungsbezogener Beratungsprozesse, auch wenn die Trägerberatung explizit genannt wird.

\section{Fortbildung in der Fachberatung}

Bislang betrachtet Fachberatung Fortbildung weitgehend als eine regionale Domäne, von der inhaltlichen, am Bedarf ausgerichteten Planung über die Erstellung von Fortbildungsprogrammen bis hin zur organisatorischen Absicherung 
der Maßnahmen. In der Vergangenheit führte dies zu einem Kaleidoskop unterschiedlichster Angebote, das sich eher am subjektiven Befindlichkeitsbedürfnis der Adressaten ausrichtete. In dessen Folge kam es und kommt es noch zu unterschiedlich motivierten und qualifizierten Angeboten, die in wenigen Fällen aus regionalen Bedingungen abgeleitet werden und wurden. Dieses System zeichnet sich zwar durch Vielfalt, weniger jedoch zwingend durch Effizienz aus.

\section{Konferenzsysteme}

Leiterinnenkonferenzen und verschiedene Arbeitskreise haben sich in der Vergangenheit als Informations- und Dialogort bewährt. Die Effizienz und der damit einhergehende Aufwand ist im Verhältnis zum erzielten Nutzen schwer einzuschätzen. Virtuelle Konferenzen bzw. virtuelle Dialoge finden bisher nur in Ausnahmen statt, obgleich in vielen Fällen die technischen Voraussetzungen gegeben sind. Gut ist der verstärkte Einsatz neuerer Methoden, z. B. Zukunftswerkstatt, World Café bis hin zu klaren, themenbezogenen Klausurtagungen.

\section{Interne Strukturen}

In Bereich der Evangelischen Kirche im Rheinland haben Fachberatungskonferenzen eine lange Tradition. Der Arbeitskreis der Fachberaterinnen ist mittlerweile in der Diakonie Rheinland-Westfalen-Lippe organisiert. Diese Konferenzen sind in den letzten Jahren vermehrt auf den Austausch gesetzlicher und betriebswirtschaftlicher Randbedingungen, bedingt durch äußere Einflüsse, reduziert worden. Dabei verloren die fachpädagogische Auseinandersetzung und die strategische gemeinsame Orientierung an Wertigkeit. Erst in jüngster Vergangenheit konnten unter anderem durch das Integrierte Bildungssystem Evangelischer Kindertageseinrichtungen (IBEK) wieder neue Impulse gesetzt werden. Dies betrifft insbesondere die regionalübergreifende Entwicklung erforderlicher Aus-, Fort- und Weiterbildungsmaßnahmen sowie die flächendeckende Einführung von Systemen zum Qualitätsmanagement.

\section{Öffentlichkeitsarbeit}

Die Öffentlichkeitsarbeit von Fachberatung sollte sehr differenziert und verschieden ausgerichtet sein. Wesentliche Zielgruppen waren für Fachberatung: die kirchlichen Gremien, politische Gremien, die Gesellschaft (von Eltern über 
das lokale Umfeld bis hin zur über-/regionalen Presse, vgl. Handbuch Fachberatung 6/2) sowie die Fachöffentlichkeit.

Hier findet eine Weiterentwicklung auf verschiedenen Ebenen statt. Die Mitwirkung in übergeordneten kirchlichen Gremien setzt ein umfassendes kirchliches Engagement voraus. Dasselbe gilt für die überregionalen politischen Ebenen, in denen es in aller Regel keine konkrete Bindung an die Tageseinrichtungen und den direkten Fachbezug gibt. Die Metaebene Fachberatung ist in den landesweiten Entscheidungsprozessen nicht involviert und wird zumindest in NRW nicht entsprechend zur Kenntnis genommen bzw. die vorliegenden Kompetenzen nicht adäquat genutzt.

Eltern sind ein wesentlicher Teil der Öffentlichkeit und somit grundsätzlich Beteiligte im frühkindlichen Betreuungs-, Bildungs- und Erziehungsgeschehen. Diese Entwicklungen werden mittlerweile von Fachberatungen berücksichtigt und entsprechend gewürdigt. Die Öffentlichkeitsarbeit via Internet wird nach und nach installiert.

Eine Orientierung am dem Selbstverständnis der Fachberatung, wie es sich in den 80er Jahren entwickelt hat und in der Verbindung zwischen Tageseinrichtungen und Träger angedacht war, ist im realen Kontext nicht mehr herzustellen. Die Beschreibung der oben genannten Prozesse ist erschwerend einhergegangen mit der Reflexion einer spätestens seit der Jahrtausendwende beginnenden Revolution in den Kindertageseinrichtungen und damit auch einer gravierenden Veränderung des Selbstverständnisses der Fachberatung.

\section{Fachberatung im Wandel}

Die aus Sicht der Fachberatung grundlegenden Dienstaufgaben werden zunehmend durch Verwaltungs- sowie durch Aufgaben überlagert, die im Rahmen zugeordneter Dienst- und Fachaufsicht entstehen. In diesem Umfeld entstehen neue Berufszuordnungen ehemaliger Fachberatung im Rheinland. Diese reichen vom pädagogischen Vorstand eines großen Trägers, der Abteilungsleitung innerhalb eines auf stadtbezogenen Trägersystems, über die pädagogische Leitung eines kleinen Verbundes bis hin zur einer neuen Ebene, die der ehemaligen Fachberatung in der neuen Funktion unterstellt ist: der Sachgebietsleitung. Umfang und Qualität dieser aktuellen Veränderungen machen deutlich, dass ein neues Berufsbild bzw. eine Rollendifferenzierung und einen eigenes Selbstverständnis mehr denn je von Nöten ist.

Der vom Sächsischen Staatsministerium für Soziales 2008 veröffentlichte Abschlussbericht „Evaluierung der Personalausstattung in Kindertageseinrichtungen sowie Struktur und Angebote der Fachberatung für Kindertageseinrichtungen in Sachsen "weist aus, dass in Sachsen 36,3\% der Fachberaterinnen folgende Aufgaben „...zusätzlich zur Fachberatung wahr(nehmen)“: allgemeine 
Verwaltungsaufgaben, finanztechnische Aufgaben, Dienst- und Fachaufsicht, berufspolitische Aktivitäten (S. 121). Diese Aufgaben besetzen etwa $50 \%$ der Arbeitszeit (siehe ebenda, S. 131).

Wenn auch die Ergebnisse nicht bundesweit verallgemeinert werden können, zeichnet sich dennoch eine deutliche Tendenz ab: Das Bild genuiner Fachberatung wird bedeutsam durch neue und andere Aufgaben überlagert, wenn nicht sogar abgelöst.

Es verwundert daher nicht, dass Fachberatung aus der Sicht des pädagogischen Personals in den Tageseinrichtungen für Kinder nur als „teilweise wirksam" erlebt wird. Zu diesem Ergebnis kommt Hense auf der Basis von 337 bundesweit zurückgesandten Fragebögen (2008, S. 136). Dort, wo sie wirkt oder wirken kann, wird Fachberatung hinsichtlich ihrer persönlichen Kompetenzen (ebenda, S. 138) gut benotet (zwischen den Schulnoten 1,8 bis 2,8), wenngleich knapp $47 \%$ das Statement „Unsere Fachberatung steht in regelmäßiger Verbindung mit den Erzieherinnen“ verneinen (ebenda, S. 142). Dieses Ergebnis darf nicht überinterpretiert werden. Befragt wurden Leiterinnen und pädagogisches Fachpersonal auf verschiedenen Ebenen und Fachberatung wirkt wahrnehmbar nicht immer auf das gesamte Personal in den Einrichtungen durch.

Fazit: Beide Studien weisen auf ein und dieselbe Problematik aus verschiedener Perspektive hin. Während die Studie in Sachsen den veränderten Operationsumfang von Fachberatung durchleuchtet, analysiert Hense (2008) die Auswirkungen verlagerter Aufgabenfelder aus der Sicht der pädagogischen Adressaten. Beide Ergebnisse machen deutlich, dass ein Wandel bzw. eine Klärung im Rollenbild von Fachberatung die Erwartungshorizonte verändern und entstandene Lücken neu gestaltet werden müssen.

\section{Fachberatung im Wandel zwischen Gestaltung und Effizienzerwartung}

Mit zunehmender Komplexität des Systems Kindertageseinrichtung wachsen auch Gestaltungs-, Steuerungs- und Koordinierungsbedarfe. Gestaltung bezieht sich dabei nicht nur auf die Entwicklung einzelner Einrichtungen, sondern insbesondere auf die strategische und finanztechnisch nachhaltige Angebots- und Infrastruktur entlang der Interessen der Umwelten, in die die Kindertageseinrichtungen eingebettet sind (z. B. Politik, Eltern, Kindeswohl). Steuerungsaufgaben, die sich im Wesentlichen aus der Fach- und Dienstaufsicht ergeben, werden dabei tendenziell stärker auf Fachberatung verlagert. Hier muss die Wechselwirkung zwischen Aufsicht und Beratung neu überdacht bzw. endlich eindeutiger geklärt oder vielleicht sogar getrennt werden. Dabei ist es nicht die Frage, ob, sondern ab welchem Beratungsanlass eine Aufsichtsfunk- 
tion und das Beratungsverständnis im Interessen- und Vertrauenskonflikt stehen können. Der Koordinierungsbedarf in Form vielfältiger Gremien- und Konferenzanlässe steigt ebenso an. In einer Voruntersuchung wurde im IBEK festgestellt, dass ca. $25 \%$ der monatlichen Arbeitszeit auf solche Arbeiten fallen können, vom Jugendhilfeverbund über Netzwerkkoordinationen bis hin zu kleinen Leitungskonferenzen und weiteren kleinteiligen, internen Konferenzsystemen (z. B. Führungskreis, Strategietagung, interne Dienstbesprechungen). Die reinen Zeitaufwendungen für die eigentlichen Tagungen bilden dabei weniger eine Herausforderung; zeitaufwendig sind die Vor- und Nachbereitungen sowie die Bewegungszeiten. So können aus drei Stunden Konferenzzeit sehr schnell acht Stunden Gesamtaufwendungen werden.

Das im vorhergehenden Abschnitt beschriebene Rollenverständnis von Fachberatung aus dem Jahr 2004 existiert in dieser Form nicht mehr. An seine Stelle ist Rollenvielfalt getreten, die sich dennoch auf wesentliche Aspekte verdichten lässt:

Zunahme der Vorgesetztenfunktion und Abgrenzung zur Beratung

1. Zunahme überregionaler Gestaltungs- und Koordinationsaufwendungen

2. Übernahme neuer Aufgaben, z. B. im Rahmen der Qualitätsentwicklung

3. Begleitung/Unterstützung in neuen fachpädagogischen Feldern

4. Zunahme der Trägerberatung und Trägerunterstützung nicht nur aus betriebswirtschaftlicher Sicht, sondern auch in Personalmanagement bis hin zur zukünftiger, strategischer und überlebensfähiger Angebotsausrichtung der Träger

Auch für Fachberatung bedeutet dies, neue Herausforderungen zuzulassen und anzunehmen sowie die dafür erforderlichen Kompetenzen zu erwerben. Wenngleich die Praxis ein attraktives Lernfeld darstellt, sind nachhaltig wirkende Weiterbildungsangebote, die sich mit der Praxis eng verzahnen, erforderlich.

Fazit: Fachberatung steht inmitten eines Systemwandels von Kindertageseinrichtungen. In diesem Wandel nimmt Fachberatung eine entscheidende Rolle ein, indem sie wesentlich umfassender eine strukturierte Personal- und Organisationsentwicklung im Dialog mit anderen Expertinnen und Experten analysiert und die dafür notwendigen Rahmenbedingungen zu sichern hilft. Stärker als in der Vergangenheit agiert Fachberatung und bringt proaktiv nachhaltige Veränderungsimpulse ein. In diesem Zusammenhang übernimmt sie verstärkt auch die Trägerperspektive.

Erst wenn die neuen Rollenprofile von Fachberatung in nächster Zeit geklärt sind, kann abschließend über Effizienz resümiert werden. Dabei sollten die Grundgedanken der Effizienz in Bezug auf die Handlungsfelder der Tageseinrichtungen für Kinder im Mittelpunkt stehen: Zentrale Aufgabe ist es nach wie 
vor, durch pädagogisches Handeln wirksam Effekte zu erzielen, die im weitesten Sinne als eine qualitativ hochwertige frühkindliche Betreuung, Bildung und Erziehung bezeichnet werden. Dies ist ein Prozess und führt zu konkreten Ergebnissen, so beherrschen z. B. alle Kinder, die in die Grundschule wechseln die deutsche Sprache in einem Umfang, der hinreicht, dem Unterricht sprachlich folgen zu können. Das Ergebnis sprachlicher Literalität erzielt Wirkungen: Beispielsweise hat ein Kind besonderen Spaß am Rechenunterricht und entwickelt sich zu einem Mathematiker, der 20 Jahre später in der Luft - und Raumfahrt arbeiten könnte. Beherrschte das Beispielkind die Sprache nicht hinreichend (Ergebnis), könnte es dem Mathematikunterricht nicht folgen und entwickelte keinen Spaß daran (Wirkung). Fachpädagogisch qualifizierte Mitarbeiterinnen erzielen somit laufend solche Ergebnisse und Wirkungen.

Aufgabe von Fachberatung ist es nach wie vor, diese Prozesse und deren Rahmenbedingungen zu unterstützen, zu begleiten, zu verbessern und zu optimieren. Die Adressaten von Fachberatung haben sich deutlich erweitert. Neben den pädagogischen Fachkräften sind Träger (Arbeitgeber) - und im Sinne eines vernetzten und systemischen Handelns - die Co-Akteure und Akteurinnen rund um die Kindertageseinrichtungen getreten.

Effizienz von Fachberatung zu beschreiben bedeutet ein erstes Annäherungsmodell zu skizzieren, weil Fachberatung sich in einem massiven Wandel befindet und der beschriebene Systemumbruch enorm ist.

Dies kann nur bedeuten, die Wirkungsfelder von Fachberatung, ob selbst angeregt oder angefragt, vor dem Hintergrund systembedingter Veränderungen aufzugreifen. Die ökonomische Wirksamkeit als Verhältnis zwischen Kosten und Nutzen (Effizienz) gelingt nur, wenn diese Handlungsfelder und Wirkungserwartungen transparent sind. Dabei orientiert sich der Beitrag an Kompetenzen, die eingebracht oder eben durch Beratung verändert werden wollen. „Kompetenzen charakterisieren die Fähigkeiten von Menschen, sich in offenen und unüberschaubaren, komplexen und dynamischen Situationen selbstorganisiert zurechtzufinden" (Heyse \& Erpenbeck (2004), S. XIII).

\section{Literatur}

Blank, U. (2009): Qualitätsentwicklung in Evangelischen Kindertageseinrichtungen. In Evangelische Kirche im Rheinland, Diakonisches Werk Rheinland e. V., Rheinische Verband e.V. (Hrsg.): Integriertes Bildungssystem Evangelischer Tageseinrichtungen für Kinder. global gedacht - lokal gemacht; 103-110

Bundesvereinigung Evangelischer Tageseinrichtungen für Kinder e.V., Diakonisches Institut für Qualitätsentwicklung im Diakonischen Werk der EKD e.V. (Hrsg.) (2009): Bundesrahmenhandbuch. Leitfaden für den Aufbau eines Qualitätsmanagementsystems in Tageseinrichtungen für Kinder 
Hense, M. (2009): Wirksamkeit der Fachberatung - eine empirische Studie. Dissertationsschrift Universität Bielefeld.

Heyse, V., Erpenbeck, J. (2004): Kompetenztraining. 64 Informations- und Trainingsprogramme. Stuttgart

König, K., Pasternak, P. (2008): elementar + professionell: Die Akademisierung der elementarpädagogischen Ausbildung in Deutschland. HoF - Arbeitsberichte 5’08. Wittenberg

Laewen, H.-J., Andres, B (Hrsg.) (2002): Bildung und Erziehung in der frühen Kindheit. Weinheim

OECD (2004): Die Politik der frühkindlichen Betreuung, Bildung und Erziehung in der Bundesrepublik Deutschland.

Rheinischer Verband Evangelischer Tageseinrichtungen für Kinder e.V. mit dem Arbeitskreis Fachberatung, (Hrsg.) (2004): QM Handbuch Evangelischer Fachberatung.

Rheinischer Verband Evangelischer Tageseinrichtungen für Kinder e. V. (Hrsg.) (2002): Hoffnung Leben. Seelze/Velber

Sächsisches Staatsministerium für Soziales (2008): Evaluierung der Personalausstattung in Kindertageseinrichtungen sowie Struktur und Angebote der Fachberatung für Kindertageseinrichtungen und Kindertagespflege in Sachsen. Abschlussbericht.

Tries,J. (2007) Early Childhood Managerin? Leitungskurse Heute - Morgen - 2027 Vortrag anlässlich der Denktage des Rheinischen Verbandes Evangelischer Tageseinrichtungen für Kinder e. V. im August 2007. Bonn

Tries, J., Walther, J. (2009): Eine veränderte Welt. In EKir, DWR, RV (Hrsg.): Integriertes Bildungssystem Evangelischer Tageseinrichtungen für Kinder. global gedacht lokal gemacht; $15-24$

Walther, J. (2009): Einführung in das IBEK. In EKir, DWR, RV (Hrsg.): Integriertes Bildungssystem Evangelischer Tageseinrichtungen für Kinder. global gedacht - lokal gemacht; $3-12$ 